\title{
Nanostructuring \\ poly-[2-methoxy-5-(2'-ethyl-hexiloxy)-p-phenylenevinylene] thin films by high-temperature soft lithography
}

\author{
Dario Pisignano $^{\mathrm{a}, *}$, Luana Persano ${ }^{\mathrm{a}}$, Francesco Babudri ${ }^{\mathrm{b}, \mathrm{c}}$, Gianluca M. Farinola ${ }^{\mathrm{c}}$, \\ Francesco Naso ${ }^{\text {b,c }}$, Roberto Cingolani ${ }^{\text {a }}$, Giuseppe Gigli ${ }^{\text {a }}$ \\ a NNL, National Nanotechnology Laboratory of Istituto Nazionale di Fisica della Materia (INFM), \\ and Dipartimento di Ingegneria dell'Innovazione, Università di Lecce, \\ via Arnesano, I-73100 Lecce, Italy \\ b CNR ICCOM, Sezione di Bari, via Amendola 173, I-70126 Bari, Italy \\ c Dipartimento di Chimica, Università di Bari, via Amendola 173, I-70126 Bari, Italy
}

\begin{abstract}
Sub micron patterning of the conjugated polymer, poly-[2-methoxy-5-(2'-ethyl-hexiloxy)-p-phenylenevinylene] (MEH-PPV) has been achieved by high-temperature soft lithography. The process has been carried out by placing a spin-coated polymer film in conformal contact with elastomeric replicas of master structures fabricated by electron beam lithography. The system is then heated to decrease the polymer viscosity, allowing the pattern transfer with resolution down to $300 \mathrm{~nm}$. The well-preserved photoluminescence spectrum and efficiency of the emissive polymer clearly indicate that high-temperature soft lithography can be successfully applied for the one-step realization of organic-based devices.
\end{abstract}

(C) 2003 Elsevier B.V. All rights reserved.

Keywords: Patterning; PDMS; Glass transition temperature

\section{Introduction}

In the last years a lot of efforts have been done to develop low-cost and flexible patterning techniques. In particular, soft lithography [1], which is based on the conformal contact between the material to be patterned and an elastomeric replica of a master structure, allows high resolution over large areas with a wide variety of compounds. Among other soft techniques, soft molding (SM) or capillary force lithography [2], joins the advantages of both soft and nanoimprint lithographies [3], as it exploits elastomeric elements in conjunction with the temperature-induced glass transition of organic compounds to transfer the pattern. In analogy with nanoimprinting, in SM lithography the mold (in this case elastomeric) is placed onto a polymeric film, and the system is driven above the glass transition temperature, $T_{\mathrm{g}}$, of the target compound. The subsequent cooling down below $T_{\mathrm{g}}$ freezes the pattern into the polymer, and the replica can be peeled off.

\footnotetext{
* Corresponding author. Tel.: +39-832-297283; fax: +39-832-326-351. E-mail address: dario.pisignano@unile.it (D. Pisignano).
}

The enhancement of capillarity effects driving the fluid polymer into the recessed features of the replica is due to the high temperature, which has been demonstrated to be a critical factor also to strongly improve the throughput of microfluidic lithography [4]. Nevertheless, operating at high temperature might limit the applicability of such technique to active organic materials, due to the possible degradation of their optical and electrical properties.

In this work, we report on the patterning by SM of a representative conjugated polymer, poly-[2-methoxy-5-( $2^{\prime}$-ethylhexiloxy)-p-phenylenevinylene] (MEH-PPV), by SM, with resolution up to $300 \mathrm{~nm}$. No significant variation of the photoluminescence (PL) spectra and of the PL absolute quantum efficiency was observed, indicating that high-temperature soft lithography can actually be considered a flexible and powerful technique for the one-step realization of organic-based devices.

\section{Experimental}

The lithography process is schematized in Fig. 1a. First, we fabricated $\mathrm{Si}$ master structures by both photo- and 


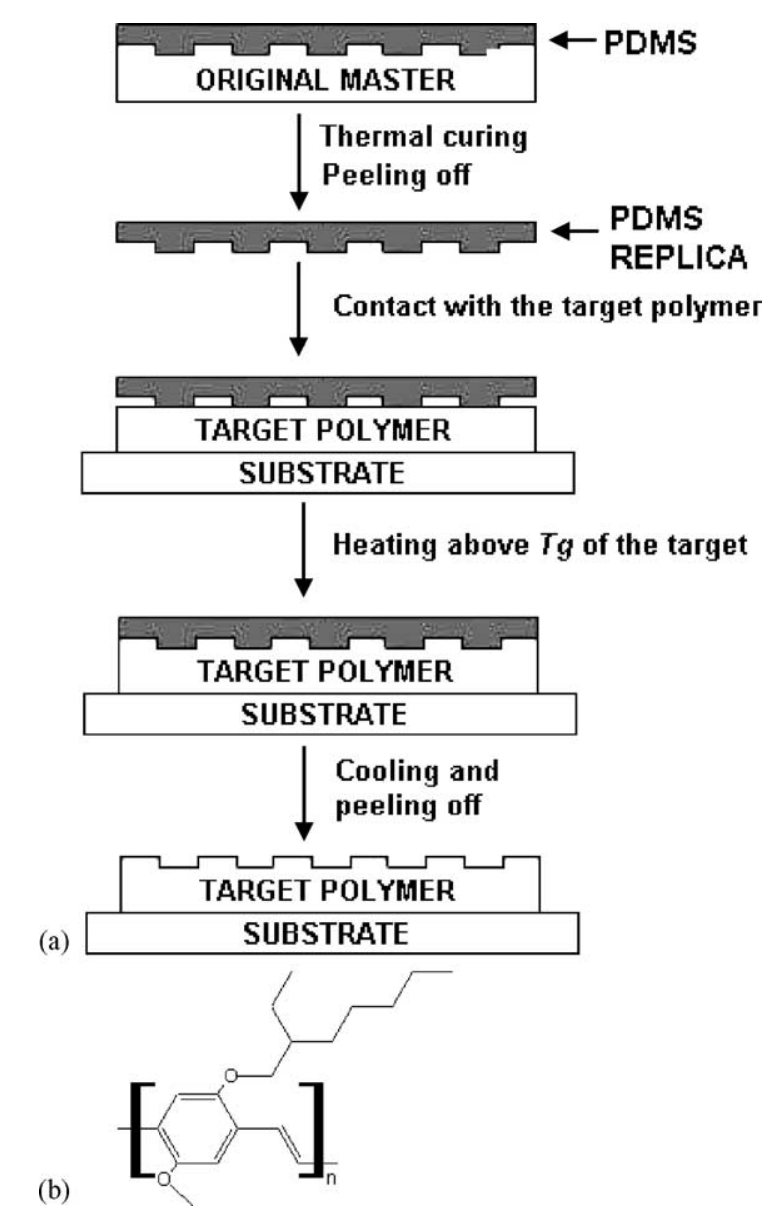

Fig. 1. (a) Schematic diagram of the process of master replication and soft molding (features not in scale). (b) Molecular structure of MEHPPV.

electron beam lithography using a Raith Elphy Plus pattern generator controlling a Leica Stereoscan 440 scanning electron microscope, working at $20 \mathrm{keV}$, followed by reactive ion etching. Then, the elastomeric replicas of the master were realized by poly(dimethylsiloxane) (PDMS, Sylgard 184, Dow Corning, Midland, MI, USA) according to a standard replica molding procedure [1], and placed onto spin-cast polymer films under their own weight. The SM lithography was carried out at $290^{\circ} \mathrm{C}$ and $10^{-3} \mathrm{~Pa}$ on spin-cast films of MEH-PPV (molecular structure in Fig. 1b), whose PL quantum yield was measured into an integrating sphere by an $\mathrm{He}-\mathrm{Cd}$ laser $(\lambda=325 \mathrm{~nm})$ [5]. The morphological investigation of the patterned film surface was carried out by optical and atomic force microscopy (AFM; NanoScope from Digital Instruments).

\section{Results and discussion}

The master pattern and its transfer to MEH-PPV are shown in Fig. 2a and b, respectively. Unlike nanoimprint

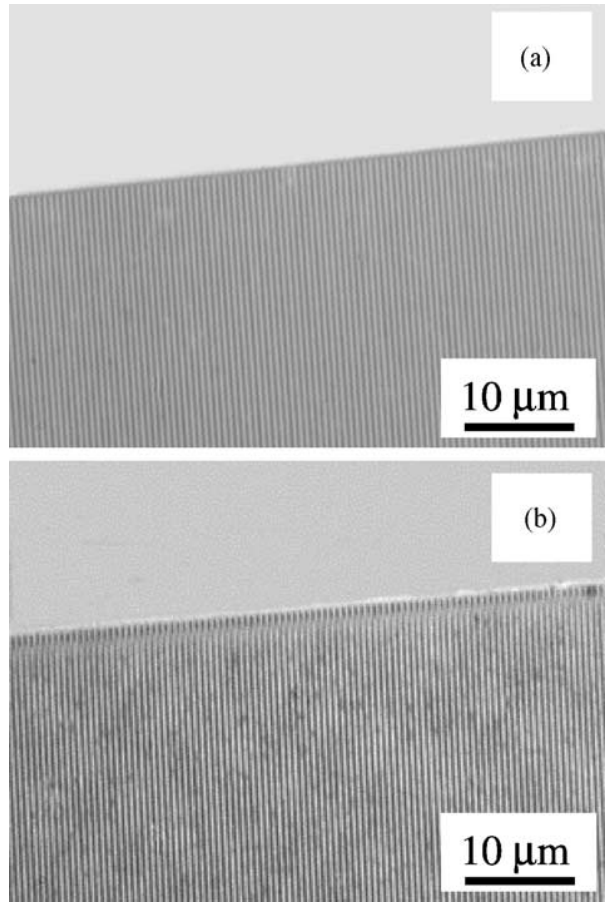

Fig. 2. Optical micrographs of a $600 \mathrm{~nm}$ period grating master (a) and SM (b) results on MEH-PPV.

lithography, which produces a negative copy of the master features on the target polymer, SM uses intermediate replicas to transfer the pattern, thus preserving the same shape (positive) of the master. SM is based on the capillarity effect, forcing the polymer to penetrate into the recessed features of the PDMS replica. Such a penetration is favored by the low polymer viscosity, as the time, $t$, needed for the filling process is given by [6]:

$t=\frac{2 \eta z^{2}}{\gamma R \cos \Theta}$

where $z$ is the feature height, $\eta$ the polymer viscosity, and $\gamma, R$, and $\Theta$ indicate the fluid-air surface tension, the hydraulic radius of the capillary, and the contact angle between the liquid and the surface of the capillary, respectively. Consequently, one can increase the temperature to exploit the strong temperature dependence of the structural dynamics of organic compounds [7], leading the viscosity to decrease because of the increased molecular mobility.

The two dimensional view and the cross section of the final polymer gratings, imaged by AFM (Fig. 3), show a well-defined pattern, whose high quality suggests that the achievable resolution can be reduced with respect to the presently obtained size, depending on the starting masters.

The PL spectra of MEH-PPV before and after SM lithography are shown in Fig. 4. Minor differences can be observed, as possible consequence of the lithography process on the films (for example the peak wavelength, $\lambda_{\max }$, 
(a)

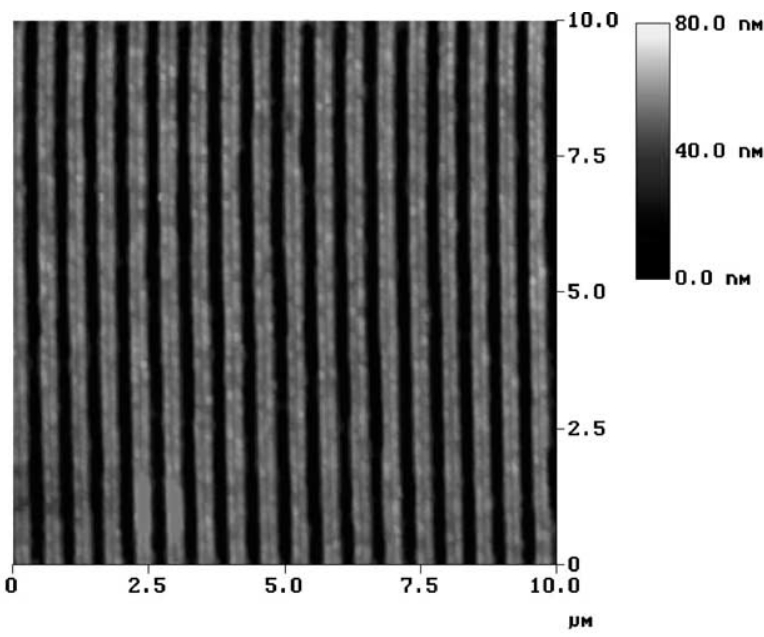

(b)

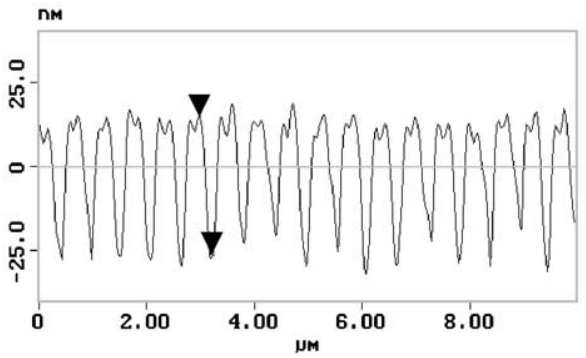

Fig. 3. AFM two $(10 \mu \mathrm{m} \times 10 \mu \mathrm{m})$ (a) dimensional view of a patterned polymer grating and corresponding cross section, (b). The height of the obtained features is about $40 \mathrm{~nm}$.

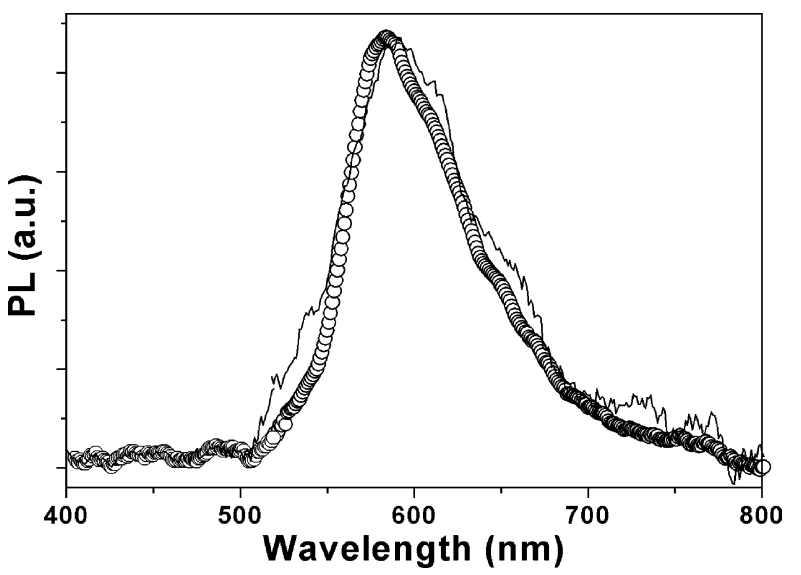

Fig. 4. MEH-PPV PL spectra before (circles) and after (line) SM lithography. exhibits a $3 \mathrm{~nm}$ red-shift after patterning). In order to have a more quantitative assessment of the retained luminescence properties of the patterned polymer, we measured the PL efficiency, which was found to be 8.0 and $7.1 \%$ before and after SM, respectively. This demonstrates that high-temperature soft lithography can actually be applied without relevant degradation of the functionality of the target materials. Finally, since the thermal-induced oxidation of vinylene groups of PPV with formation of carbonyl moieties is strongly affected by the atmosphere [8], experiments of SM under higher vacuum conditions are currently in progress in our laboratory to further improve the process and preserve the native PL quantum yield of the polymer.

\section{Conclusion}

In conclusion, we have shown that SM lithography can be effectively applied to light-emitting and gaining conjugated polymers, thus allowing the immediate realization of organic-based opto-electronics devices. A number of issues of SM need to be further investigated, including its ultimate resolution and the maximum achievable aspect ratio, which could be limited by distortions and collapse of soft PDMS structures. Nevertheless this technique is undoubtedly very promising for organic-based micro- and nanofabrication.

\section{References}

[1] Y. Xia, G.M. Whitesides, Angew. Chem. Int. Ed. 37 (1998) 550.

[2] K.Y. Suh, Y.S. Kim, H.H. Lee, Adv. Mater. 13 (2001) 1386.

[3] S.Y. Chou, P.R. Krauss, P.J. Renstrom, Appl. Phys. Lett. 67 (1995) 3114.

[4] D. Pisignano, E. Sariconi, M. Mazzeo, G. Gigli, R. Cingolani, Adv. Mater. 21 (2002) 1565.

[5] N.C. Greenham, I.D.W. Samuel, G.R. Hayes, R.T. Phyllips, Y.A.R.R. Kessener, S.C. Moratti, A.B. Holmes, R.H. Friend, Chem. Phys. Lett. 241 (1995) 89.

[6] D. Myers, Surfaces, Interfaces and Colloids, Wiley, New York, 1999.

[7] K.L. Ngai, D.J. Plazek, in: J.E. Mark (Ed.), Physical Properties of Polymers Handbook, American Institute of Physics Press, Woodbury, New York, 1996.

[8] F. Papadimitrakopoulos, K. Konstadinidis, T.M. Miller, R. Opila, E.A. Chandross, M.E. Galvin, Chem. Mater. 6 (1994) 1563. 